\title{
X-ray magnetic circular dichroism and reflection anisotropy spectroscopy Kerr effect studies of capped magnetic nanowires
}

\author{
J. P. Cunniffe ${ }^{1}$, D. E. McNally ${ }^{1}$, M. Liberati ${ }^{2}$, E. Arenholz ${ }^{2}$, C. McGuinness ${ }^{1}$, and J. F. McGilp ${ }^{*}, 1$ \\ ${ }^{1}$ School of Physics, Trinity College Dublin, Dublin 2, Ireland \\ ${ }^{2}$ Advanced Light Source, Lawrence Berkeley National Laboratory, Berkeley, California 94720, USA
}

\begin{abstract}
Aligned Co wires grown on $\mathrm{Pt}(997)$ under ultra-high vacuum conditions have been capped successfully by the epitaxial growth of $\mathrm{Au}$ monolayers (ML) at room temperature. The samples were kept under vacuum except when transferring between apparatus or when making some of the measurements. No degradation of the Co wires was detected during the measurements. The magneto-optic response of the system was measured using $\mathrm{x}$ ray magnetic circular dichroism (XMCD) at the Co $L_{2,3}$ edge and reflection anisotropy spectroscopy (RAS) at near-normal-incidence, which is sensitive to the normal component of the out-of-plane magnetization via the Kerr
\end{abstract}

effect (MOKE). Capping the wires significantly impacts their magnetic properties. Comparison of the magnetooptic response of the system at $\mathrm{x}$-ray and optical energies reveals small differences that are attributed to the induced moment in the Pt substrate and Au capping layer not picked up by the element specific XMCD measurements.. The sensitivity of RAS-MOKE is sufficient to allow the determination of the easy axis direction of the capped wires to within a few degrees. The results for a 6atom-wide Co wire sample, capped with $6 \mathrm{ML}$ of $\mathrm{Au}$, are consistent with the capped wires possessing perpendicular magnetization.
1 Introduction Magnetic nanostructures exhibit novel physical phenomena not observed in bulk magnetic systems [1]. The magnetic properties of these lowdimensional systems often depend sensitively on both the morphology and symmetry of the nanostructures and the substrate on which they are grown [2]. Magnetic nanostructures must be protected from the environment if they are to have a technological application and this is often accomplished by capping the structure with a thin nonmagnetic layer. However, capping these structures with protective layers of non-magnetic material may modify their magnetic properties [3, 4], making the characterization of the buried interfacial region of such a capped structure essential to understanding the underlying physics and materials science. Conventional surface techniques are insensitive to buried nanostructures, but a variety of $\mathrm{x}$ ray and optical techniques, with their larger penetration depth, can provide useful data. X-ray spectroscopies can give information on shallow buried layers where their elemental sensitivity and, in the case of x-ray magnetic circular dichroism (XMCD), their magnetic sensitivity [5], can provide some of the desired information [6]. However, x-ray spectroscopies generally require synchrotron radiation sources for these experiments. In and around the visible region, special optical techniques ("epioptics"), using conventional broad band and laser sources, have been developed that exploit the difference in the optical response of the interface and bulk. Epioptic techniques can be used to measure the optical response of buried nanostructures with sub-monolayer (ML) resolution [7].

Deposition of Co on clean Pt(997) between $250 \mathrm{~K}$ and $300 \mathrm{~K}$, under ultra-high vacuum (UHV) conditions, results in the self-organized growth of periodic arrays of atomic wires along the upper edge of the monatomic steps of the (997) surface [8, 9]. The width of the terrace allows aligned wires from one to seven atoms wide to be grown by controlling the coverage, making it a very useful model system for probing magnetism in atomic scale nanowires. 
The effect of capping on the magnetism of this model system has not yet been reported and, in this paper, the magneto-optical response of capped cobalt nanowires is explored in the visible and $\mathrm{x}$-ray regions of the electromagnetic spectrum.

2 Experiment and phenomenology The samples were prepared under UHV conditions with a base pressure below $5 \times 10^{-11}$ mbar. The $8 \mathrm{~mm}$ diameter vicinal $\operatorname{Pt}(997)$ single crystal was cleaned by cycles of Ar ion etching and annealing until a sharp $(1 \times 1)$ low energy electron diffraction (LEED) pattern appeared and no residual contamination was observed with Auger electron spectroscopy (AES) Cobalt was evaporated onto the clean $\operatorname{Pt}(997)$ surface at room temperature (RT) using a high temperature Knudsen cell, the deposition rate having been calibrated using AES and a quartz crystal oscillator. AES showed no detectable contamination of the Co layers at the end of the deposition process. Gold capping layers were deposited onto the substrate at RT using a second high temperature Knudsen cell. LEED showed a sharp $(1 \times 1)$ pattern after depositing up to $9 \mathrm{ML}$ of $\mathrm{Au}$, indicating epitaxial growth. An eclipsing mask was used during deposition to produce a checkerboard pattern comprising 3-wire and 6-wire patches capped by 3, 6 and $9 \mathrm{ML}$ of $\mathrm{Au}$, where each patch can be separately probed. No degradation of the magnetic properties was observed during the short exposure to atmospheric pressure required by some of the measurements.

$\mathrm{X}$-ray absorption spectra (XAS) and x-ray magnetic circular dichroism (XMCD) spectra of the Co $L_{2,3}$ edges were obtained by recording the total electron yield as a function of x-ray energy. The high-resolution monochromator had a resolving power of 7000 at $800 \mathrm{eV}$. Left and right elliptically polarized $\mathrm{x}$-rays were used with a degree of circular polarization $P_{c}= \pm 0.9$ from an elliptically polarizing undulator on beamline 4.0.2 at the Advanced Light Source [10]. Magnetic fields of up to $\pm 500 \mathrm{mT}$ were applied along the x-ray propagation direction using an eightpole electromagnet [11] with field switching between positive and negative field values at each photon energy value. Measurements were taken at room temperature and at $80 \mathrm{~K}$ The crystal was mounted with the wires aligned parallel to the axis of rotation of the manipulator ( $y$ axis), allowing the response in the $x z$ plane orthogonal to the wire direction to be probed, where $z$ is the surface normal. Hysteresis loops were measured by monitoring the Co $L_{3}$ intensity while ramping the field and reversing the polarization after each cycle. The asymmetry is then calculated as the difference of Co $L_{3}$ intensity. The observed XAS and XMCD spectra are shown in Fig. 1 where the XMCD spectrum is defined as

$$
X M C D=I_{-}-I_{+}
$$

where $I_{+}\left(I_{-}\right)$is the total electron yield when the photon angular momentum is parallel (anti-parallel) to the fieldinduced magnetization (Fig. 1).
The reflection anisotropy spectroscopy (RAS) system has been described previously [12]. Briefly, RAS measures the difference in reflectance, at near normal incidence, of light linearly polarized in two orthogonal directions at the surface plane of a cubic material [13]:

$$
\frac{\Delta r}{r}=2 \frac{r_{x}-r_{y}}{r_{x}+r_{y}}
$$

For ferromagnetic materials, RAS can probe the normal component of the magnetization via the polar magneto-optic Kerr effect (MOKE) [14]. The complex Kerr angle for an ultrathin magnetic layer of thickness $d$, magnetooptic constant $Q$, and dielectric function $\varepsilon$ is given by

$$
\phi_{\mathrm{Kerr}}=i \frac{r_{+}-r_{-}}{r_{+}+r_{-}}=\frac{2 \omega d Q}{c} \frac{\varepsilon}{\varepsilon_{S}-1}
$$

where $r$ are the complex reflection coefficients for the photon angular momentum defined previously, $\omega$ is the frequency of the light, and $\varepsilon_{S}$ is the dielectric function of the non-magnetic substrate. The RAS-MOKE rotation can be found from the difference in the real part of the RAS response when the magnetization direction is reversed [14]. This spectroscopic technique, which uses a high frequency photoelastic modulator and phase-sensitive detection, offers a sensitivity comparable to single wavelength laser based MOKE systems.

Hysteresis loops are fitted using a simple sigmoidal function

$$
M^{ \pm}(H)=-M_{\text {sat }}+2 M_{\text {sat }} /\left\{1+\exp \left[-s\left(H \mp H_{c}\right)\right]\right\}
$$

where $M^{+}(H)$ has the applied magnetic field $H$ increasing from an initial negative value, and conversely for $M^{-}(H), M_{\text {sat }}$ is the saturation magnetization, $s$ is the softness, and $H_{c}$ the coercivity [15]. A four-pole electromagnet provided magnetic fields up to $\pm 100 \mathrm{mT}$ for loop measurement. In addition, a constant field could be rotated in the $x z$ or $y z$ planes for measuring the direction of the easy axis of magnetization.

3 Results and discussion Capping by Au at RT appears to leave the nanowire structure undisturbed. The XAS at the Co $L$-edge shown in Fig. 1 are characteristic of metallic Co with neither a pre-peak, nor a shoulder at the $\mathrm{Co} L_{3}$ edge, which would indicate Co-O bonds. Both AES and the absence of oxidation of the Co on exposure to atmosphere confirm that the Co does not segregate to the surface. The RAS-MOKE spectra measured immediately after growth have remained unchanged over a period of months indicating that the sample has remained protected by the capping layer [16]. In addition, capped 2-, 3- and 4atom wide wires show oscillatory behaviour in their coercivity, similar to that observed for uncapped wires [17].

Figure 1 (upper) shows the RT XMCD total electron yield spectra for 6-atom-wide Co wires (0.78 ML) on $\mathrm{Pt}(997)$, capped with $6 \mathrm{ML} \mathrm{Au}$. The photon angular mo- 
mentum is parallel $\left(I_{+}\right)$or anti-parallel $\left(I_{-}\right)$with respect to the applied field, at an angle $\theta=0^{\circ}$ in the $x z$ plane relative to the surface normal. Figure 1 (lower) shows the resulting XMCD signal, i.e. difference of the two XAS spectra obtained for opposite fields. Figure 2 shows XMCD hysteresis loops where the magnetic field vector is aligned collinearly with the x-ray wavevector, and the angle of incidence is varied in the $x z$ plane orthogonal to the wires. Good sigmoidal fits are obtained when $180^{\circ}$ domain wall motion is assumed to be the dominant mechanism of magnetization reversal, where the effective field is the component of the applied field along the easy axis [18]. The capped 6-atom-wide Co wires have a coercivity $H_{c}=15 \pm 1 \mathrm{mT}$ and a softness $s=1.3 \pm 0.1$. Capping by 6 ML of Au has reduced the coercivity of the system significantly: uncapped 4-atom-wide $\mathrm{Co}$ wires have $H_{c} \sim 320 \mathrm{mT}$, while $1.3 \mathrm{ML}$ of $\mathrm{Co}$ on $\mathrm{Pt}(997)$ has $H_{c} \sim 220 \mathrm{mT}$ [17]. Close inspection of Fig. 2 shows a small difference between the $\pm 45^{\circ}$ plots, indicating that the easy axis is not exactly normal to the surface.

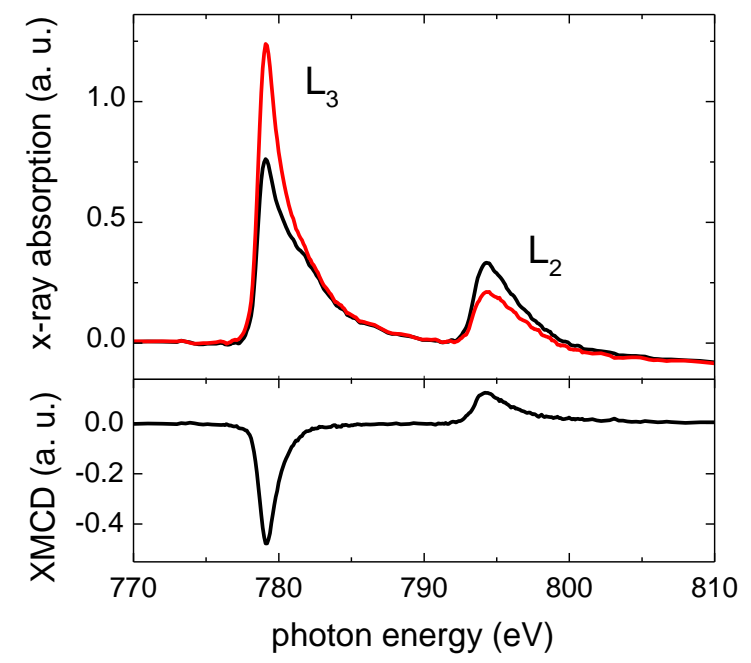

Figure 1 Upper: RT XMCD total electron yield spectra at $\theta=0^{\circ}$ for 6-atom-wide Co wires on $\mathrm{Pt}(997)$, capped with $6 \mathrm{ML}$ $\mathrm{Au}$; the photon angular momentum is parallel (一) or anti-parallel $(-)$ to the field-induced magnetization. Lower: XMCD asymmetry.

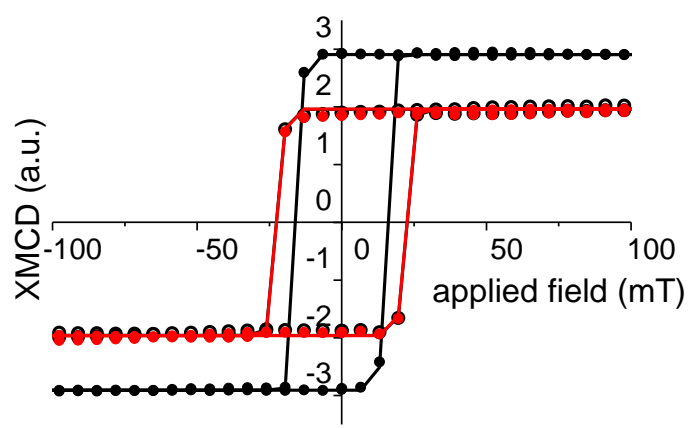

Figure 2 RT XMCD hysteresis loops for the 6-atom-wide Co wire sample capped with $6 \mathrm{ML}$ of Au. The magnetic field vector is aligned with the $\mathrm{x}$-ray wavevector and the angle of incidence in the $x z$ plane orthogonal to the wires is varied by rotation of the sample about the $y$ axis; solid lines are fits to the data: $\theta=0^{\circ}$ 一), $\theta=+45^{\circ}(\mathrm{o},-), \theta=-45^{\circ}(\bullet,-)$.

RAS and RAS-MOKE spectra are shown in Fig. 3. No significant optical anisotropy is observed in the visible region, while the RAS-MOKE signal shows an increase towards the UV. A good signal/noise ratio is available around $2 \mathrm{eV}$, allowing hysteresis loops and easy axis measurements to be made at this energy.

Figure 4 compares XMCD and RAS-MOKE loops for normal incidence and it can be seen that the RAS-MOKE loop is a little wider and softer, with $H_{c}=18 \pm 1 \mathrm{mT}$ and $s=0.28 \pm 0.05$. It appears likely that, in this spectral region, the RAS-MOKE loops are recording the total magnetic response of the system, including spin-polarization effects in the substrate and capping layer, while the XMCD loops, being element specific, are recording only the response of the Co wires. It is well known that an induced polarisation of Au capping layers occurs due to the interaction with the magnetic Co [19]. This observation, combined with the much reduced coercivity from either uncapped 4-atom wide wires or 1.3ML of Co on Pt(997) [17], is indicative that the Au capping has indeed greatly modified the magnetic anisotropy of the system, as has been observed previously in capped Co spherical clusters [4, 20, 21]. However, unlike the previous observations which were for spherical particles, the capping can be seen to induce a preferred orientation for the easy axis of magnetization. 

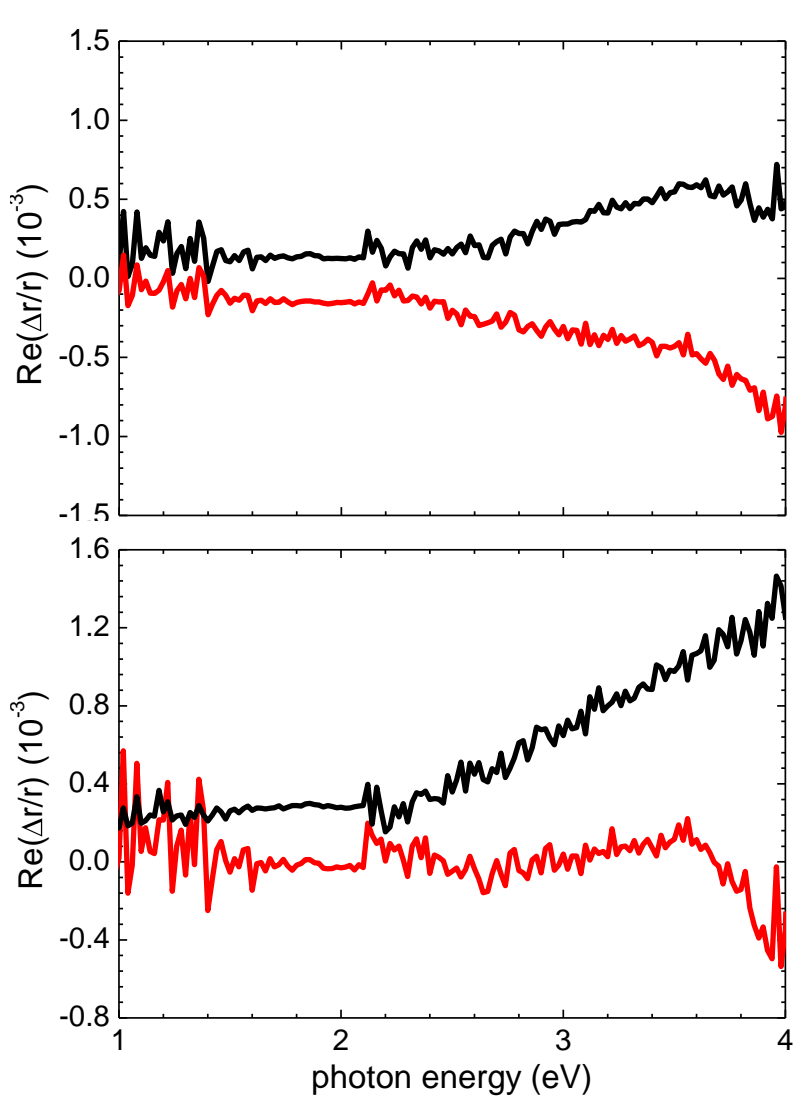

Figure 3 RAS spectra for the 6-atom-wide Co wire sample capped with $6 \mathrm{ML}$ of Au. Upper: for positive (-) and (-) negative saturation magnetization. Lower: the sum $(-)$ and difference (一) of the upper curves. The difference is the RAS-MOKE signal.

A simple method of easy axis determination has been developed that involves measuring the RAS-MOKE response while rotating a constant applied field about the surface normal (Fig. 5). As discussed above, the effective field for magnetization reversal is the component of the applied field along the easy axis. Fitting to Eq. 4 is then limited by the strong correlation between the coercivity and the easy axis direction. This can be removed by recording scans for clockwise and anti-clockwise rotation, where the coercivity contribution changes sign. For field rotation in the $x z$ plane orthogonal to the wires, simultaneous fits to the two curves gives $H_{c}=17 \pm 1 \mathrm{mT}$ and $s=0.35 \pm 0.05$, with an easy axis direction of $-8^{\circ} \pm 3^{\circ}$. For the $y z$ plane, the results are $H_{c}=17 \pm 1 \mathrm{mT}$ and $s=0.35 \pm 0.05$, with an easy axis direction of $2^{\circ} \pm 3^{\circ}$. The terraces of $\operatorname{Pt}(997)$ are at $-6.45^{\circ}$ to the surface normal, leading to the conclusion that the easy axis of magnetization is along the normal to the $\operatorname{Pt}(111)$ terraces and perpendicular to the capped 6-atom-wide Co wires, to within experimental error. The XMCD results of Fig. 2 are consistent with the small departure of the easy axis from the surface nor- mal. The interface magnetocrystalline anisotropy is known to play a principal role in the perpendicular magnetic anisotropy observed in ultrathin film magnetic multilayers [22]. In the present system all the Co atoms reside in the interfacial region and it is not unexpected that perpendicular magnetic anisotropy of the Co wires is observed, stabilised by the electronic and magnetic interaction at the interface between the $\operatorname{Pt}(997)$ substrate, the Co wires and the Au capping layer.

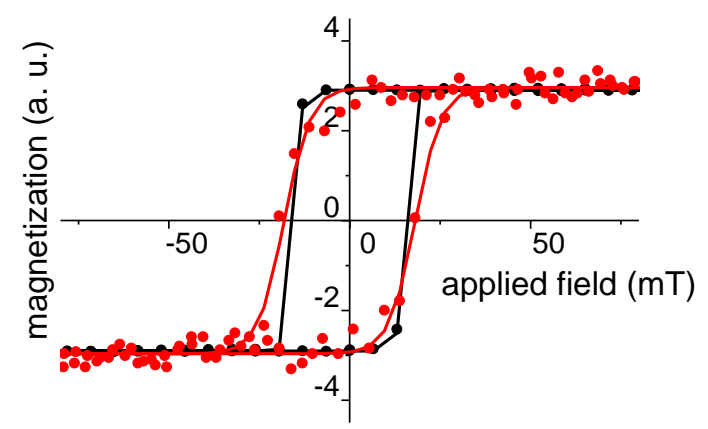

Figure 4 Comparison of RT hysteresis loops at normal incidence for the capped 6-atom-wide Co wire sample. The magnetic field vector is aligned with the photon wavevector: XMCD data and fit $(\bullet,-)$, RAS-MOKE data and fit $(\bullet,-)$, at $2.0 \mathrm{eV}$.

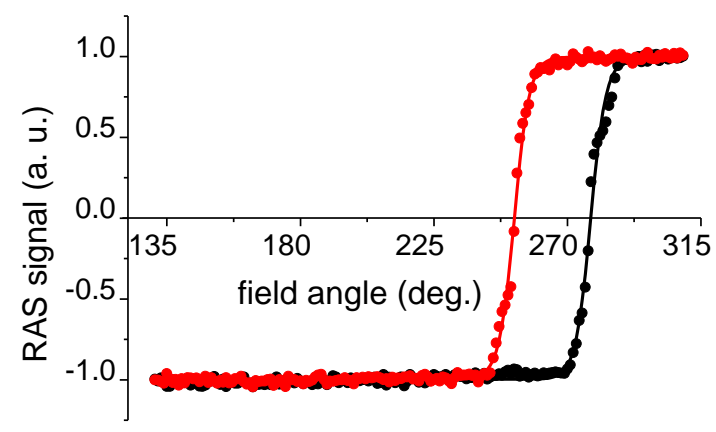

Figure $5 \mathrm{RT}$ RAS response at $2.0 \mathrm{eV}$ for the capped 6-atomwide Co wire sample, with an applied field of $73 \mathrm{mT}$ rotated in the $x z$ plane orthogonal to the wires. Clockwise rotation, data and fit $(\bullet,-)$; anti-clockwise rotation, data and fit $(\bullet,-)$.

4 Conclusion Aligned Co wires grown on $\operatorname{Pt}(997)$ have been capped successfully by the epitaxial growth of $\mathrm{Au}$ monolayers at room temperature. Capping the wires produces a large change in their magnetic properties. Comparison of the magneto-optic response of the system at 
$\mathrm{X}$-ray and optical energies reveals small differences that are attributed to the element-specific nature of XMCD. The sensitivity of RAS-MOKE for this system is sufficient to allow the easy axis direction of the capped wires to be determined to within a few degrees. The results are consistent with the capped wires possessing perpendicular magnetization along the $\mathrm{Pt}(111)$ direction.

Acknowledgements This publication has emanated from research conducted with the financial support of Science Foundation Ireland. The ALS is supported by the U.S. Department of Energy under Contract No. DE-AC02-05CH11231.

\section{References}

[1] F. J. Himpsel, J. E. Ortega, G. J. Mankey, and R. F. Willis, Adv. Phys. 47, 511 (1998).

[2] S. D. Bader, Surf. Sci. 500, 172 (2002).

[3] W. Weber, D. Kerkmann, D. Pescia, D. A. Wesner, and G. Guntherodt, Phys. Rev. Lett. 65, 2058 (1990).

[4] J. Bartolome, L. M. Garcia, F. Bartolome, F. Luis, R. Lopez-Ruiz, F. Petroff, C. Deranlot, F. Wilhelm, A. Rogalev, P. Bencok, N. B. Brookes, L. Ruiz, and J. M. Gonzalez-Calbet, Phys. Rev. B 77, 184420 (2008).

[5] J. Stohr, and H. C. Siegmann, Magnetism - From Fundamentals to Nanoscale Dynamics (Springer-Verlag, Berlin, 2006), Vol. 152, pp. 820.

[6] K. Amemiya, S. Kitagawa, D. Matusmura, T. Yokoyama, and T. Ohta, J. Phys.: Condens. Matter 15, S561 (2003).

[7] J. F. McGilp, J. Phys.: Condens. Matter, in press (2009).

[8] P. Gambardella, A. Dallmeyer, K. Malti, M. C. Malagoli, W. Eberhardt, K. Kern, and C. Carbone, Nature 416, 301 (2002).

[9] P. Gambardella, J. Phys.: Condens. Matter 15, S2533 (2003). [10] A. T. Young, E. Arenholz, F. Jun, H. Padmore, S. Marks, R. Schlueter, E. Hoyer, N. Kelez, and C. Steier, Surf. Rev. Lett. 9, 549 (2002).

[11] E. Arenholz, and S. O. Prestemon, Rev. Sci. Instrum. 76, 83908 (2005)

[12] K. Fleischer, L. Carroll, C. Smith, and J. F. McGilp, J. Phys.: Condens. Matter 19, 266003 (2007).

[13] P. Weightman, D. S. Martin, R. J. Cole, and T. Farrell, Rep. Prog. Phys. 68, 1251 (2005).

[14] T. Herrmann, K. Ludge, W. Richter, K. G. Georgarakis, P. Poulopoulos, R. Nunthel, J. Lindner, M. Wahl, and N. Esser, Phys. Rev. B 73, 134408 (2006).

[15] L. Carroll, K. Fleischer, J. P. Cunniffe, and J. F. McGilp, J. Phys.: Condens. Matter 20, 265002 (2008).

[16] L. Gan, R. D. Gomez, C. J. Powell, R. D. McMichael, P. J. Chen, and W. F. Egelhoff, J. Appl. Phys. 93, 8731 (2003).

[17] P. Gambardella, A. Dallmeyer, K. Maiti, M. C. Malagoli, S. Rusponi, P. Ohresser, W. Eberhardt, C. Carbone, and K. Kern, Phys. Rev. Lett. 93, 077203 (2004).

[18] J. J. Becker, J. Appl. Phys. 38, 1015 (1967).
[19] F. Wilhelm, M. Angelakeris, N. Jaouen, P. Poulopoulos, E. T. Papaioannou, C. Mueller, P. Fumagalli, A. Rogalev, and N. K. Flevaris, Phys. Rev. B 69, 220404 (2004).

[20] F. Luis, F. Bartolome, F. Petroff, J. Bartolome, L. M. Garcia, C. Deranlot, H. Jaffres, M. J. Martinez, P. Bencock, F. Wilhelm, A. Rogalev, and N. B. Brookes, Europhys. Lett. 76, 142 (2006). [21] F. Luis, J. Bartolome, F. Bartolome, M. J. Martinez, L. M. Garcia, F. Petroff, C. Deranlot, F. Wilhelm, and A. Rogalev, J. Appl. Phys. 99, 08 G705 (2006).

[22] D. Weller, Y. Wu, J. Stohr, M. G. Samant, B. D. Hermsmeier, and C. Chappert, Phys. Rev. B 49, 12888 (1994). 